V.

\title{
Welches sind die Bedingungen für die Spontanheilung der Zuckerkrankheit?
}

Von

Prof. Dr. HEINRICH BENEDICT $\uparrow$,

dir. Chefarzt der inneren Abteilung des israelitisehen Krankenhauses in Budapest.

Das im Titel genannte Problem ist eigentlich eine Umkehrung des Problems der Diabctespathogenese. Es handelt sich nämlich um die Frage: Mil welchen Mitteln erreicht es der Organismus, daß die Zuckerjrankheit in einem nicht unbeträchtlichen Teile der Fälle von selbst ausheilt? Wie das Studium des Heilungsverlaufes der Infektionskrankheiten auch die Pathogenese beleuchtete und für die Möglichkeit einer rationellen Behandlung manchen wertvollen Fingerzeig lieferte, halte ich es nicht für ausgoschlossen, daß Forschungen in der angegebenen Richtung $\mathrm{zu}$ theoretischen und praktischen Resultaten führen, die auch für den Therapeuten bedeutungsvoll sein können. Ein Problem ist nur das ,Wie?" der spontanen Heilung. Daß ein nicht geringer Teil der Zuckerkranken im Laufe der Jahre langsam seine Glykosurie verliert und seine Kohlenhydrattoleranz wieder das normale Niveau erreicht, ist eine allgemein bekannte Tatsache. Es handelt sich nicht um vorübergehende Glykosurien infolge toxischer, nervöser oder infektiöser Einflüsse, sondern um Personen, die aus zuckerlkranken Familien stammen, auf der Höhe ihres Lebens unter den charakteristischen klinischen Symptomen erkrankt sind, die sich Jahre hindurch in ihrer Stoffwechselstörung, in ihrer Reaktion auf diätetische Maßregeln von anderen Zuckerkranken nicht unterscheiden and die gewöhnliche diätetische und balneologische Therapie ebenso lässig, vielleicht noch lässiger durchgeführt haben, wie ihre Kollegen in Saccharo. Und doch konstatieren wir bei ihnen den regressiven Charakter der Krankheit statt jenes progressiven Verlaufes, welchen die klassischen Schilderer der Zuckerlzrankheit für den, ,echten " Diabetes postulieren. Mag sich auch diese Heilung manchmal nur in einer konstant bleibenden Normalisierung der Kohlenhydrattoleranz äußern, so 
ist dooh die Zahl der Kranken groß, die jahre- und jahrzehntelang in praktischem Sinne als diabetesfrei zu betrachten sind. Man kann vielleicht sogar sagen, daß der regressive Charakter der Zuckèrkrankheit bei der in späteren Jahren erworbenen Form die Regel, der progressive Charakter die Ausnahme bildet. Denn auch dort, wo der Kranke seiner ursprünglichen Zuckerkrankheit zum Opfer fällt, handelt es sich nicht um den immer fortschreitenden, zu Azidose und Koma führenden Zusammenbruch des Stoffwechsels, sondern um Organveränderungen, welche mit der Zuckerkrankheit nur mittelbar zusammenhängen and welche fortschreiten können, während die Stoffwechselstörung in den Hintergrund tritt oder erlischt.

Doch selbst für den Diabetes der Jugendlichen muß die Tuehre von der ständigen Progression im Charakter der Krankheit revidiert werden. Schon vor der Insulinära war es gelungen, die durch Bourchardat, Dïring, Kolisch, Allen, Falta und andere propagierte kalorienarme, besonders eiweißarme Diät manchen bösartig scheinenden, im Zeichen der Progression stehenden Diabetes zur Rückbildung zu bringen, vielleicht hie und da auch zu heilen.

Nun hat aber die Praxis außer der spontanen Heilungsmöglichkeit der Zuckerkrankheit auch die unwiderlegliche Tatsache erbracht, daß diese Spontanheilung fast ausnahmslos an das Auftreten gewisser krankhafter Zustände gebunden ist, welche sich entweder aus der Zuckerkrankheit selbst entwickelten oder mit dieser parallel einhergingen oder unabhängig von ihr auftraten. Dem unbefangenen Beobachter mußte sich die U̇berzeugung aufdrängen, daß eine andere Krankheit die Zuckerkrankheit verdrängt oder abgelöst habe, so daß nicht nur Ärzte, sondern auch sich selbst und ihre Mitkranken ständig beobachtende intelligentere Zuckerkranke die Besserung oder Zurückbildung der Glykosurie manchmal argwöhnisch beobachten.

Unsere erste Aufgabe wäre demnach eine Aufzählung derjenigen pathologischen Zustände, welche erfahrungsgemäß zum Schwinden der Glykosurie führen können, die weitere Aufgabe die Erforschung derjenigen pathophysiologischen Faktoren, welche im Verlaufe derselben eine der Entwicklung des Diabetes entgegengesetzte Stoffwechsellage schaffen.

Die wichtigsten pathologischen Zustände, welchen ich bei meinen eigenen Kranken und in der Literatur begegnete, sind: die Leberzirrhose, die chronisch verlaujende Nierenschrumpfung, 
die mit der allgemeinen Arteriosklerose einhergehende Entkräftung, die allgemeine senile Entkräftung ohne auffallende Arteriosklerose, bei Männern die Prostata-Hypertrophie mit ihren Folgen, die Karzinose und ausnahmsweise die Tuberkulose. Auf den ersten Blick mag es erscheinen, daß diese Zustände alle auf eine vereinfachte, gemeinsame Formel zurïckzuführen sind, so daß man einfach sagt, daß die fortschreitende Kachexie des Kranken die Zuckerkrankheit zum Schwinden bringt, doch ist diese Vereinfachung nichts weniger als eine Erklärung. Mögen sich auch die meisten Lehr- und Handbücher mit einer solchen begnügen, so weist doch z. B. schon Naunyn in seiner klassischen Monographie darauf hin, daß bei diesen Kranken eigentlich eine Steigerung der Kohlenhydrattoleranz besteht, welche sich sicherlich nicht in den Rahmen der gewöhnlichen Kachexie-Definition einfügen läßt. Ist doch überdies der Begriff der Kachexie auch noch ein ungeklärter; ihre pathologisch-anatomischen und pathologischphysiologischen Komponenten müssen je nach der auslösenden Krankheit, ja selbst nach der Individualität des befallenen Kranken wechseln.

Schon die klassischen Autoren der Zuckerkrankheit haben darauf hingewiesen, daß die Ausbildung einer Leberzirrhose diese antagonistisch beeinflusse. Vor der Entdeckung des Panlsreasdiabetes suchte man unter dem Einflusse der Untersuchungen Glaude Bernards die Ursache der Zuckerkrankheit in der Leber, und wenn heute wieder die zentrale Rolle der Leber im normalen und pathologischen Zuckerstoffwechsel fester gegründet erscheint als je, so wissen wir doch, daß die anatomischen Veränderungen dieses Organes selbst bei den schwersten Fällen von Diabetes äußerst selten sind, wie auch anderseits die schwersten anatomischen Lebererkrankungen nicht zum Diabetes führen. Doch beschrieb bereits Claude Bernard einen Kranken, der beinahe Jahrzehnte hindurch große Zuckermengen ausgeschieden hatte, später aber an Leberzirrhose erkrankte und die Zuckerkrankheit vollständig verlor. Er schließt dem Obduktionsbefunde die Worte an: „Kein Wunder, vielmehr natürlich, daß die gesteigerte Zuckerproduktion der Leber in das Gegenteil übergeht, sobald das Lebergewebe schwindet." Lépine beobachtete ähnliche Fälle.

Persönlich erinnere ich mich an zwei. 1 . Als ich vor 12 Jahren meine jetzige Krankenhausabteilung übernahm, fand ich dort einen 62 jähr. Gastwirt mit Leberzirrhose vor, welcher dort in den Jahren vorher mehrmals mit mittelschwerem Diabetes in Behandlung gestanden hatte. Mit der Ausbildung der Zirrhose war er aber vollständig zuckerfrei geworden. 
Seine Zuckertoleranz unterschied sich nicht von derjenigen des Gesunden. Thre Besserung ging ungefähr mit der Ausbildung der Zirrhose parallel. Neben dem Anzeichen typischer portaler Stauung zeigte der Kranke überdies mäßige Abmagerung, normale Zirkulationsorgane und Nierenfunktion. Auf die Talmaoperation, während welcher sich der tastende Finger des Chirurgen von der körnigen Leberschrumpfung überzeugen. konnte, ging die Bauchwassersucht zurück. Der Kranke prësentierte sich Jahre hindurch von Zeit zu Zeit im. Spitale ohne Ascites und mit ganz normaler Zuckertoleranz.

2. Ich war zu einer 54jährigen Patientin mit schwerer Lingenentzündung pro consilio gerufen. Bei der Untersuchung fiel die dunkle Bronzefarbe der Patientin auf, ferner ihre 4 Finger uiber den Nabel reichende, äußerst voluminöse, harte und glatte Leber und die große Milz. Ich erfuhr, daß die Kranke in dem 35. Jahre zuckerkrank wurde und ungefähr 10 Jahre hindurch Karlsbad besuchte. Nach den Protokollon war dio Glykosurie eine beträchtliche gewesen und hatte manchmal $7 \%$ ïberschritten. Sie hatte nie strenge Diät gehalten and sich außerhalb ihrer Karlsbader Kuren. nur auf die Vermeidung von Zueker beschränkt. Num ist sie seit einigen. Jahren zuckerfrei, doch hat sie seitdem das Anschwellen. ihres Oberbauches und die Braunfärbung ihrer Haut beobachtet. Die Kranke blieb auch. während und nach der Pneumonie vollständig zuckerfrei.

Es ist überaus zweifelhaft, ob in diesen Fällen das Schwinden der Zuckerkrankheit unmittelbar von der Leberzirrhose abhing. Man könnte sich zwar vorstellen, daß cine kompensatorische Hyperplasie des spezifischen Lebergewebes, wie sie bei der typischen Laennecschen Zirrhose regelmäßig statthat, den Organismus langsam in den Besitz eines Leberparenchyms gelangen läBt, welches zur Glykogenstapelung wieder geeignet ist. Doch ist dies unwahrscheinlich. Denn auch die übrigen Leberfunktionen pflegen sich nicht mit dem Fortschreiten der Zirrhose zu bessern. Näherliegend wäre die Annahme, daß ein Teil der Kohlenhydrate infolge der erschwerten portalen Zirliulation teils auf dem Wege venöser Kollateralen, teils auf dem Lymphwege mit Umgehung: der Leber in den allgemeinen Stoffwechsel gelangt sei. Wie die gute Verwertung des intravenös oder rektal gegebenen Zuokers beim Diabetiker beweist, wären die Verwertungsmöglichkeiten des ,ahepatisch" in den Organismus gelangten Zuckers günstigere. Doch werden alle diese Annahmen überflüssig angesichts der Tatsache, daß in diesen Fällen die Lebererkrankung gewöhnlich einer Pankreaszirrhose parallel geht. Ebenso wie die Zuckerkrankheit durch die Pankreasaffektion verursacht war, müssen wir auch die Bedingungen der spontanen Heilung in erster Linie im Pankreas suchen. Dies gilt ebenso für die gewöhnliche atrophische Leberzirrhose, wie für den Bronzediabetes, welcher in 
unserem zweiten Falle zweifellos bestand. Ist es doch sicher, daß das anatomische Substrat des letzteren gleichfalls eine interstitielle Entzündung des Pankreas ist.

Nach der heutigen Auffassung sind die Pankreasinseln der. Bildungsort des spezifischen Hormons. Es ist noch ungewiß, ob zwischen diesen Inseln und dem sezernierenden Pankreasparenchym morphologisch und funktionell scharfe Grenzen gezogen werden können. Doch wenn wir auch mit der Mehrzahl der pathologischen Anațomen annehmen, daß diese Inseln sich im postembryonalen Leben unter verschiedenen pathologischen Einwirkungen aus. dem azinösen Gewebe und den Ausführungsgängen des Pankreas neu bilden können, so ändert es doch nichts an der Wahrscheinlichkeit, daß die Inseln der Schatplatz der inneren Hormonbildung sind. Hingegen ist es wichtig, daß diese Inselneubildung sowohl im Tierversuche als auch in der menschlichen Pathologie dort am intensivsten war, wo der Schwund des azinösen Pankreasparenchyms am ausgesprochensten in Erscheinung trat.

Nach Herxheimer bilden sich die Inseln aus Parenchym, sio verlieren ihre äußere Sekretion, während sich die innere steigert. Moldenhauer fand, daß nach Unterbindung der Ausführungsgänge bei ausbleibendem Diabetes. die Inseln intakt blieben und außerdem das schwindende Parenchym sich zu Inseln umgestaltet. Hansemann entfernte im Tierversuche das Pankreas stückweise und konnte in den Ùberresten eine Auflösung der azinösen Struktur, eine Gruppierung der Zellen um die Blutgefäße und endlich eine Vermehrung der Inseln beobachten. Die Glykosurie ist in diesen. Fällen meistens vorübergehend und tritt manchmal auch bei maximaler Kohlenhydratbelastung nicht wieder auf. Die anatomischen Erfahrungen Fahrs stimmen mit diesen experimentellen Daten überein. Bei der Selktion von 26 Diabetikern, unter denen 11 an Koma, I5 an anderen Erkrankungen. gestorben waren, fand er $20 \mathrm{mal}$ die Umwandlung des azinösen Pankreas. gewebes zu Inseln. Auch ihm scheint die Inselneubildung aus den Ausführungsgängen wahrscheinlich. Allen unternahm ähnliche histologische Untersuchungen auf sehr breiter Basis und kam zu gleichem Resultate; er kommt so zu der Feststellung, daß Zuckerkrankheit eigentlich keine progressive Stoffwechselerkrankung sei.

Neuerdings hat sich Hoppe-Seyler mit diesen Fragen beschäftigt. Seine Untersuchungen beziehen sich in erster Linie auf ältere Individuen, clie an interstitieller Pankreatitis oder Granularatrophie auf sklerotischer Basis litten. Er fand die Inselregeneration fast regelmäßig. Bei einer früher luetischen Iranken z. B., die lange an Diabetes und Furunkulose gelitten hatte, hörte dio Glykosurie langsam auf. Sie ging an einer Influenza zugrunde, und man fand bei der Sektion als wahrscheinliches Substrat des gebesserten. Kohlenhydratstoffwechsels neben den zugrunde gegangenen Gewebepartien hypertrophische, ganze Nester bildende Inseln. Diese Neubildung war besonders in den kaudalen Pankreaspartien ausgeprägt. 
Rhodenburg beobachtete einen ähnlichen. Fall. Eine Frau litt bis zu ihrem 40. Jahre an Zuekerkrankheit. Mit der Menopause verschwand die Glykosurie. 5 Jahre später kam sie mit chronischer Nephritis und Herzschwäche in die Klinik. Nach dem Tode fand man im Pankreaskopfe fibröse Entartung, doch im Pankreaskörper starke Inselneubildung; die Schilddrüse war fibrös degeneriert. Dieser Fall bildet also einen Übergang zu clenjenigen, in welchen die Spontanheiling der Zuckerkrankheit nicht nur von der Noubildung der Pankreasinseln, sondern auch von den Veränderungen anderer Organe abhängt. Auf diese soll später eingegangen werden. Svale Vincent stellt es geradezu als Regel hin, daß alles, was zur Pankreasatrophie führt, jede Kachexie, ja selbst der protrahierte Hunger, die Hyperplasie des Inselapparates zur Folge hat.

Nun halte ich es aber für wahrscheinlich, daß die Atrophie des Pankreasparenchyms auch unabhängig von der Inselhyperplasie einen wichtigen Faktor bei der Regressivitität der Zuckerkrankheit bildet. Die Kohlenhydrattoleranz ist im allgemeinen um so größer, je größer der Gesamtstoffwechsel des Organismus ist. Ebenso steht die Intensität der Zuckerkrankheit mit der Größe des Eiweisstoffwechsels in geradem Verhältnisse.

Früher bestand die allgemeine Ansicht, daß sowohl der Gesumt-, als auch der Eiweißstoffwechsel bei den Zuckerkranken naturgemäß gesteigert sei. Heute müssen wir die Feststellung amerikanischer Stoffwechselforscher akzeptieren, daß wenigstens in den späteren Stadien der Krankheit der Gesamtstoffwechsel des Zuckerkranken im Vergleiche zu Individuen mit ähnlichem Körpergewichte und ähnlicher Körperoberfläche verringert sei. Auch kann man den Stickstoffwechsel Zuckerkranker, d. h. die N-Menge, bei welcher der Zuckerkranke noch im Eiweißgleichgewicht bleibt, nach Allen, Maignon, Pelrén und anderen bei entsprechend fettreicher, aber $\mathrm{N}$-armer Ernährung noch tiefer herabdrücken und leichter Stickstoffansatz erzielen, als bei Gesunden. Die zu erwartende Steigerung der Stickstoffausscheidung bei Eiweißzulagen, welche deh Ausdruck der in der Leber vonstatten gehenden Desamidierung bildet, ist weniger lebhaft. Die Voraussetzungen für das Herabgehen des Gesamtstoffwechsels müssen wir im ganzen Organismus suchen, d. h. in der Körpermasse, den peripherischen Zellen ebenso wie in dem Zustande der endokrinen Drüsen und des vegetativen Nervensystems; die Voraussetzungen für den herabgeminderten Stickstoffumsatz finden wir aber in erster Linie in der herabgesetzten Leberfunktion. Nun ist wieder deren Abhängigkeit von der Magen- und Pankreasverdaunung, welche die ersten Phasen der Eiweißdissimilation beherrschen, sehr leicht möglich. Es ist wahr, daß man mit der 
Duodenalsonde nur in einem geringen Teile der Fälle ein Absinken der äußeren Pankreasselzretion nachweisen konnte. Doch haben, wie oben erwähnt, die anatomischen Untersuchungen gerade bei älteren Personen ein Herabgehen des Pankreasgewichtes fast auf die Hälfte nachgewiesen. Nehmen wir noch hinzu, daß der größte Teil der älteren Zuckerkranken an Achylia gastrica leidet, so können wir uns vorstellen daß die schon im Darmkanale eintretende Verlangsamung des Eiweißabbaues auf den Fortgang der intrazellulären Eiweißdissimilation hemmend einwirkt, was wieder auf die Kohlenhydrattoleranz von günstigem Einflusse sein kann. Ich berufe mich auf meine seinerzeit mit $N$. Roth angestellten Versuche, welche eine starke Abhängigkeit der Stickstoffausscheidungskurve vom Zustande des Magens nachwies. Bei Hyperaziden finden wir nach einer eiweißhaltigen Probediät eine rasche und steile Nitrogenausscheidung, welche bei Achylie langsamer verlief und bei Pankreasachylie fehlte.

Bei jedem Zuckerkranken, der nicht in zweckwidriger Weise mit Eiweiß übernährt wird, tritt die fallende Tendenz des endogenen Eiweißstoffwechsels im Laufe der Z̈eit ein, und gleichzeitig mit ihm heben sich die Chancen der Kohlenhydrattoleranz. Der wichtigste exogene Faktor dieses herabgesetzten EiweiBstoffwechsels ist die Eiweißbeschränkung der Nahrung, d. h. man muß darauf bedacht sein, die Eiweißzersetzung nicht künstlich anzufachen. Unter den endogenen hemmenden Faktoren stehen neben den erwähnten regressiven Veränderungen des Pankreas, der Leber und vielleicht der Magenschleimhaut, die endokrinen Organe wohl in erster Linie. Hier kommt die wichtigste Rolle der Schilddrüse zu. Seit der Entdeckung des Insulins ist die Bodeutung der übrigen diabetogenen Organe ein wenig in den Hintergrund gerückt, doch sind auch für den Fall, daß im Zentrum des Kohlenhydratstoffwechsels das Pankreas steht, die Korrelationen der Bauchspeicheldrüse mit den übrigen endokrinen Organen, in erster Linie mit der Schilddrüse für den Gang der Erkrankung im Einzelfalle verantwortlich zu machen.

Ich will nicht die Frage aufwerfen, ob es einen primären Diabetes infolge Schilddrüsenhyperfunktion gibt, doch ist es möglich, daß eine gesteigerte Schilddrüsentätiglzeit eine solche Gesamtstofîwechsel- und Eiweißstoffwechsellage zeitigt, neben welcher eine sonst genügende Insulinproduktion ungenügend wird. Denken wir serner an den Antagonismus, welcher nach Eppingers, Faltas und Rudingers noch immer nicht widerlegten Untersuchungen Arehiv für Verdaungskrankheiten. Bd, XI. Hoft 34. 
zwischen der inneren Sekretion des Pankreas und der Schilddrüse besteht. Wine herabgesetzte Insulinproduktion zieht eine Șteigerung der Schilddrüsentätigkeit nach sich. Bei den akut einsctzenden Zuckerkrankheiten, welche im Beginn noch mit einer Kalorienüberproduktion und mit gesteigertem Eiweißstoffwechsel einhergehen, läßt sich letztere durch eine Steigerung der. Schilddrüsentätigkeit am leichtesten erklären. Tch könnte eine ganze Reihe von Kranken aufzählen, bei welcher der Ausbruch der Zuckerkrankheit gleichzeitig mit einer Basedowerkrankung: erfolgte. Noch zahlreicher sind die Fälle mit hyperthyreotischen Symptomen. Wir müssen sagen, daß die Sohwellung der Schilddrüse, die Labilität des Herz- und GefäBsystems, das Gräfesche. Symptom, der Tremor, besonders bei den Diabetikerinnen der 40 er und 50er Jahre die Regel bilden, wenn die Franen gleich zu Beginn der Krankheit in unsere Beobachtung kommen.

Gibt es aber klinische Beobachtungen und verläBliche anatomische Befunde, aus welchen wir bei regressivem oder ausheilendem Diabetes auf eine herabgeselzle Schilddrüsenfunktion schließen können? A priori scheint diese Annahme kaum von der Hand zu weisen. Bei den in diesem Stadium beobachteten Kranken fiel stets eine Atrophie der Schilddrüse auf, gleichviel, ob es sich um Kranke mit allgemeinem Marasmus, Arteriosklerose oder chronischer Nephropathie handeite, außerdem ihre Hypothermie, ihre herabgesetzte vasomotorische Reizbarkeit, ihre Odembereitschaft. Systematische pathologische Untersuchungen der endokrinen Organe bei geheilten Diabetikern st shen nosh aus. Das verhältinismäBig große Material, welches E. Kraus in Prag aufarboitete ohne sichere GesetzmäBigkeiten im histologischen Befunde der Schilddrüse, der Hypophyse und der Nebennieren aufzufinden, ist für die von uns gestellte Frage nicht von Belang, weil dort die regressiven oder geheilten Diabetilker keine besondere Kategorie bilden. Rhodenburg fand in dem bereits zitierten Falle von geheiltem Diabetes fibröse Entartung der Schilddrüse. A hnlliche Befunde werden von Friedemann und Gottesmann mitgeteilt. Interessant sind ihre Tierversuche. Sie konnten bei Hunden den durch Pankreasexstirpation hervorgerufenen Diabetes in die benigne, dem fetten Diabetes der Menschen analoge Form überführen, wenn sie auch einen Teil der Schilddrüse exstirpierten. In einem Teile der Fälle ging die Zuckerkrankheit ganz zurück.

An meiner Abteilung Jxonnte ich folgende Fälle beobachten. 1. 51 jühr. Frau. Ein Jahr nach der Menopause. Herzklopfen, Atembeschwerden, all- 
gemeine Erregbarkeit, Schlaflosigkeit. Bei der Aufnahme $86 \mathrm{~g}$ Zucker im Tagesurin, reichlich Azeton, stark positiver Gerhardt. Symmetrische, weiche, nicht pulsierende Struma, Exophtalmus. Tremor, Puls 132, Blutdruck 155, extrasystolische Arhythmie, das Herz in allen Richtungen erweitert, linke Kammer auch hypertrophisch, Stauungskatarrh, beginnende Stauungsleber. In 6 Wochen gehen bei eiweißarmer Diät $(6,5 \mathrm{~g}$ N.) 28 Kalorien auf $1 \mathrm{~kg}$ Körpergewicht, die Zuckertoleranz auf $60 \mathrm{~g}$, gleichzeitig gehen die thyreotoxischen Symptome langsam zurück. In den letzten 2 Wochen kommt die Cewichtsabnahme, welche seit Beginn der Erkrankung $6 \mathrm{Isg}$ betragen hatte, zum Stillstande. Mit den Diätvorschriften der letzten Woche sucht sie einen Flöhenkurort auf, von welchem sie nach 6. Wochen in vollständig verändertem Zustande zurïckkehrt. Trotzdem ihre Kalorienaufnahme in der dortigen Kuranstalt nicht höher war als in unserem Krankenhaus, hat das Törpergewicht um $8 \mathrm{~kg}$ zugenommen. Zucker ist auch nach Genuß von $300 \mathrm{~g}$ Brol nicht mehr nachzuweisen. Die thyreotoxischen Symptome sind geschwunden. Puls 72, Blutdruck noch immer etwas erhöht. Spitzenstoß leicht hebend, sonst am Herzen nichts abnormes; Schilddrüse halb so groß wie früher, von viel härterer Beschaffenheit. Nur der Exophtalmus ist bestehen geblieben, ja er hat sich mit doppelseitiger Abduzenslähmung kompliziert. Untersuchung des Grundumsatzes am Kroghschen Apparate ergibt eine Herabsetzung von 12\%.

2. Bei einem 17 jühr. Mittelschüler tritt die Zuckerkrankheit in Begleitung klassischer Basedowsymptome auf, bloß der Exophtalmus ist nicht entwickelt. Es handelte sich um eine nodöse Struma mit selkundärer Basedowifikation. Die Entzuckerung gelingt nur, nachdem wir ihn 2 Wochen hindurch mit äußerst eiweißarmer Diät ernährten. Bei Gemüsefettcliät komnten wir den Stickstoffumsatz des $48 \mathrm{~kg}$ schweren. Kranken auf $4,2 \mathrm{~g} \mathrm{~N}$. herabsetzen und gleichzeitig die Zuckertoleranz auf $160 \mathrm{~g}$ Weißbrot. heben. Zu unserer UUberraschung hörte der Gewichtsssturz mit dem Rückgang der thyreotoxischen Erscheinungen nicht nur auf, sondern das Gewicht stieg auch an, ohne daß wir mehr als 27 Kalorien pro Kilogramm Körpergewicht gereicht hätten. Die Schilddrüse ging vor unseren Augen stark zurück. Hier konnte von Heilung nicht die Rede sein, doch ist es auffällig, wie günstig der Rückgang der Schilddrüsenhyperfunktion den Verlauf der Zuckerkrankheit beeinflußt hatte.

3. Bei einer 53 jähr. Frau, die seit Krankheitsbeginn in meiner Behandlung steht, trat die Zuckerkrankheit vor 14 Jahren unter eigentïmlichen Symptomen auf. Es zeigte sich Hämoptoe, leichte Infiltration der rechten Lungenspitze, gleichzeitig Anschwellung der Schilddrüse mit thyreotoxischen körperlichen und nervösen Begleiterscheinungen sowie Hypertonie. Einige Wochen später wurde Zucker im Urin festgestellt. Der Diabetes war stets ein schwerer. Zuckerfreiheit war 10 Jahre hindurch nur vorübergehend zu erzielen. Azeton und Azetessigsäure geben beständig zu schaffen. Jedle Verschlimmerung der Zuckertoleranz wurde durch ein. stärkeres Hervortreten der thyreotoxischen Erscheinungen und der hypertonischen Beschwerden eingeleitet, doch reagierte sie auch in den günstigen Perioden auf jede Kohlehydratzulage mit Glykosurie.

Brom und Antipyrin unterstützten die Behandlung günstig. Im Winter 1924 Insulinbehandlung; bei 40 Einheiten konnte man die Toleranz 
kaum bis zu $50 \mathrm{~g}$ Weißbrot steigern. Schon damals zeigte sich otwas Albumen im Urin. Im Sommer 1925 machte sie eine langwierige febrile Thrombophlebitis der linken Vena cruralis durch, die sich 2 Monate lang mit entzündlichen Lungenlkomplikationen hinzog. Die Ernährung war' eine kalorien- und eiweißarme. Überdies erhielt sie währond der ganzen Krankheit täglich 30 Einheiten Insulin. Nach Ablauf dor Krankheit und Abbruch der Insulinzufuhr war der Zucker aus dem Urin vollständig geschwunden, bloß bei $300 \mathrm{~g}$ Weißbrot zeigte sich dieser in Spuren. Hingegen führt der Urin 3-6\% Eiweiß, hie und da rote Blutkörperchen, das spezifische Gewicht fixiert sich auf 1011. - Bei Gomïsefottlkost $1,7 \mathrm{~g} \mathrm{~N}$ im Urin. Der Blutzucker beträgt 0,35-,05\%; am auffallendsten aber ist absolute Rǚclcbildung der Schilddrüse, die überhaupt nicht melur zu tasten ist, der nervösen Überregbarkeit und der Augensymptome. Dor Rest $\mathbb{N}$ erhöht sioh auf $120 \mathrm{mg}$.

In diesem Falle mögen, ähnlich wie in dem Falle Rhodenburgs, die Nephropathie und der Schilddrüsenschwund als glykosuriebeschränkende Faktoren miteinander konkurrieron, doch möchte ich letzterer die entscheidende Rolle zuerkennen.

Es ist zweifellos, daß in diesen Fällen, aber vichleicht bei jedem Zuckerkranken, die eiweißarme Diät als natürliche Bremse der Schilddrüsenhyperfunktion entgegenwirkt. Die Erfahrungen der Kriegsjahre haben gezeigt, daß die Kalorien-, besonders aber fleischarme Diät die Zahl der Basedowerkrankungen herabsetzte und den Zustand mancher Kranken besserte. Wir haben den „Eiweißschaden" bei der Basedowkrankhoit kennen gelemt und deren Diät einer Revision unterzogen, nachdem wir wissen. daß die zur Synthese des Thyroxins notwendigen Bausteine in gewissen aromatischen Gruppen des Eiweißes enthalten sind, Es ist wohl denkbar, daß dasjenige, was wir bei den Zuckerkranken "Eiweißschaden" nennen, zum großen Teile seinen Weg über die Hyperfunktion der Schilddrüse nimmt. Halten wir den Zuckerkranken bei eiweißarmer Diät, so kann seine Schilddrüsenfunktion langsam in das Gegenteil übergehen; Kalorien- und Sticlsstoffumsatz sinken unter die Norm, und gleichzeitig erhöht sich seine Zuckertoleranz so weit, daß man im gegebenen Falle praktisch von einer "Heilung" der Zuckerkrankheit sprechen kann.

Bei den äbrigen endokrinen Organen bewegen wir uns noch weit mehr im Reiche der Hypothese.

Ob das Nebennierensystem und die Hypophyse im späteren Stadium der regressiven Zuckerkrankheit ihre Tätiglkeit gleichfalls herabsetzen und so einerseits zur Hemmung der hepatischen Zuckermobilisierung, anderseits zur Hemmung des allgemeinen Stoffwechsels führen, war noch nicht Gegenstand systematischer 
Untersuchungen. Bezüglich der Nebennieren scheint manches dafür zu sprechen. Bei den Kranken mit anfangs gesteigerter Schilddrüsentätigkeit findet sich gleichzeitig häufig arterieller Hochdruck, gesteigerte Adrenalinempfindlichkeit, welche mit ersterer parallel wieder absinkt. Bei Kranken mittlerer Jahre kann eine Zuckerkrankheit, welche unter Blutdrucksteigerung, Blutgefäßkrisen, Koronarkrämpfen in Erscheinung trat, in Besserung, ja selbst in Heilung übergehen, wenn diese offenbar durch Sympathikusüberreizung veranlaßten Symptome sich milde $n$. oder verschwinden. Besonders auffällig wird dies, wenn mit dem. Nachlassen der Koronarkrisen der Herzmuskel insuffizient wird.

Ich sah in meiner Privatpraxis zwei Zuckerkranke nach.7- bzw. 10 jährigem Bestande der Krankhoit auf diese Weise ihren Zucker verlieren. Bei beiden entstand die Glykosurie zwischen dem 50. bis 60 . Jahre, bei beiden fiel im Laufe der Jahre der anfangs stark erhöhte Blutdruck be* deutend ab. In einem Falle leitete paroxysmales Herzflattern Jahre hindurch die Schwächung des Herzmuskels ein; nach dreijähriger Zuckerfreiheit (Blutzucker $0,16 \%$ ) trotz freier Diët trat wieder Zucker in geringen Mengen auf (7-8 $\mathrm{g}$ täglich). Gleichzeitig entwickelte sich eine Phthise, der die Kranke in 4 Monaten erlag.

Es ist schon den ältesten Beobachtern aufgefallen, daß beim Fortschreiten chronischer Nephropalhien, besonder, bei Nephrosklerose, die Glykosurie aufhört und die Kohlenhydrattoleranz ansteigt. Diese Fälle bilden das größte Kontingent der sich als geheilt betrachtenden Zuckerkranken. Eine Grundbedingung für das Auftreten der Glykosurie ist die normale ,Durchlässiglkeit" der Nieren für Zucker, und eines der ersten Zeichen herabgesetzter Nierenfunktion kann es bekanntlich sein, daß diese Durchlüssigkeit abnimmt. Dies sehen wir am besten bei Prostatikern. Is kommt vor, daß eine seit Jahren bestehende Glykosurie aufhört, wenn die durch die Prostatahypertrophie bedingte Harnstaung: zur Schädigung der Nierenfunktion führt. In diesen Fällen löst die Pollakisurie infolge Blasendistension die diabetische Polyurie unmerklich ab, der Urin bleibt weiter reichlich und hell, der Kranke durstet, trinkt und uriniert viel, doch zeigt die chemische Untersuchung Zuckerfreiheit und niedriges spezifisches Gewicht, so daß es den Anschein hat, als ob sich die Zuckerkrankheit in einen Diabetes insipidus gewandelt hätte. Wenn sich bei entsprechender konservativer oder operativer Behandlung die Nierenfunktion bessert, kann der Zucker wieder erscheinen. Tch habe geradezu den Eindruok gewonnen, als ob manche Zuckerkrankheit. durch ein mit aszendierender Harnstauung verbundenes Blasenleiden in dauernder Latenz gehalten werden kann. 
Ein 65jähr. Mann stand seit 3 Jahren unter meiner Beobachtung. Mit 30 Jahren hatte er oine gonorrhoische Striktur aquiriert, die so vernachlässigt wurde, daß sich im Laufe der Jahre eine hochgradige Stautungsblase und Stauungsnierenbecken entwickelte. Bei andanernder Katheterbehandlung chronische Infëktion der Harnwege. Der Kranke hatte stëndigr einen milchig getrübten Flarn vom spez. Gewicht 1009; viel Eitor und etwas mehr Eiweiß, als diesem entsprach, keine ronalen Formelemente, niemals Zucker. Starker Durst, aber absolute Appetitlosigkeit; sporadisches Temperaturerhöhung, starke Abmagerung. Im. Blute 63 mg Rest -N. Da der Kranke keinen Dauerkatheter vertrug, wurdo eine Dpizystostomie gemacht und ein Pezzer Katheter eingelegt. Dor Kranke fiihlte sich vorerst erleichtert. 5 Wochen später suchte er mich wieder auf, lor Appetit; habe sich auffallend gebessert, er esse wieder sehr viel, miisse noch immer viel trinken, doch sei er noch immer sehr schwach. Das spezilisoho Gowichti war 1016. Ich war überrascht über dio schnelle Besserung der Nierenfunktion und dachte nicht an Zucker. 12 Tage später wurlo ich mit dom behandelnden Arzte zusammen zu dem Kranken berufen. Wr bofund sich im ausgesprochenen diabetischen Koma. Der Harnzuokor botrug 7,3\%, der Blutzucker 0,64\%, der Rest-N 0,78\%. Trotz intensivstor Insulinbehandlung erfolgte am nüchsten Abend der Tod.

Wenn sich ein wirkliches chronisches Nierenloiden boin Zuckerkranken entwickelt, so bleibt der Zuckergehalt des Bhtess nicht bloß auf der früheren Höhe, sondern steigt meistens an. Bisher galt der gesteigerte Zuckergehalt des Blutes nicht für einen Faktor, welcher zu einer Besserung der diabetischen Stoffwechselstörung führen kann; gilt es doch, abgesehen vom Niorendiabetes, als ausgemacht; daB die erste Bedingung der Glyliosurie die Hyperglykämie sei.

Nehmen wir nun an, daß das entscheidonde Moment beim Diabetes die gesteigerte Zarkerbildung in der Leber ist, so macht die Niere dadurch, daß sie das Blut zum größten Teile von seinem Zuckerüberschusse befreit, deì Weg für den Weitertransport aus der Leber wieder frei.

Sehen wir aber das Wesen der Zuckerkrankheit in der verminderten Verbrauchsfähigkeit der Peripherie, so läßt sich die Sache derart formulieren, daß beim Diabetiker der AusscheidungsSchwellenwert des Zuckers für die Niere niedriger ist als diejenige Schwelle, bei welcher die Zellelemente des Körpers den Zuckes. aufzunehmen und zu verwerten imstande sind. All dies kehrt sich um, wenn die Niere für den Zucker weniger durchlïssig wird. Schon v. Noorden erklärte die Besserungstendenz nierenleidender Zuckerkranker damit, daß das gleiche Zuckermolekül jetzt länger im Organismus lreist und endlich dennoch der Zuckerzersetzung anheimfällt. Wie immer auch die Sache steht, 
soviel steht fest, daß das verstärkte Zuckerangebot unter gewissen Umständen auch im diabetischen Organismus die Zuckerverwertung steigern kann. Bestes Beispiel dafür ist die gute Verwertung des intravenös gegebenen Zuckers; die gute Wirkung der Kohlenhydrattage, besonders nach vorhergehender Karenz, ist dergestalt zu erklären, daß bei plötzlicher Exhöhung des Blutzuckerspiegels die Zuckerschwelle der peripherischen Zellen frither erreicht wird als die Zuckerschwelle der Niere. Ebenso, wie man beim sogenannten ,Wassersto ${ }^{\prime \prime}$ die kranke Niere dazu zwingen kann, daß sie die plötzlich angebotene große Flüssigkeitsmenge auf Kosten der wasserfixierenden Gewebekolloide an sich reiBt und ausscheidet, ebenso kann man clie Gewebselemente durch den plötzlichen ,ZuckerstoB" in die Lage bringen, daß sie den Zucker auf Kosten der Niere in sich aufnehmen. Die Zuckerschwellendifferenz zwischen Nieren und Geweben, welche wir beim normalen Diabetiker durch gewisse Maßregeln vorübergehend zugunsten der Gewebe beeinflussen können, ist bei dem nierenkranken Diabetiker ständig gegeben.

Ahnliche Gedankengänge finden sich bei Ambard und seinen Mitarbeitorn. Indem er das Wirkungsgesetz der Diastasenphänomene der Zuckerverbrennung anwendet, kommt er zu folgenden. zwei Schlußfolgerungen: 1. Die Verwertung der Glukose steht in geradem Verhältnisse zur Insulinämie, wenn die übrigen Faktoren, besonders der Zuckergehalt des Blutes und der Gewebe gleich sind. 2. Die Verwertung der Glukose steht in geradem Verhältnisse zur Glykämie, wenn der Gehalt des Blutes an Insulin der gleiche ist. Bei einer gegebenen Insulinproduktion muß die Verwertung mit der Glykämie steigen. Ambard hat es sogar vorgeschlagen, die Hyperglykämie des Diabetikers als ein kompensatorisches Element für die gestörte Zuckerverbrennung zu betrachten.

Bezüglich der ,Zuckerschwelle" soll festgestellt werden, daß es sich hierbei um komplexe Vorgänge der Zuckerpermeabilität und Zuckerfixierung in den Zellen handeln muß. Lichlwitz and Schittenhelm haben neuestens darauf hingewiesen, daß zwischen diesen Prozessen in der Leber und den Körperolomenten einerseits, in den Nieren anderseits ein gegensätzliches Verhalten bestehen dürfte; das Insulin hat auch einen renalen Faktor, indem es die Fixierung des Zuckers in den Nierenzellen und infolgedessen auch seine Ausscheidung hemmt. - Eine diffuse Erkrankung der Niere führt aber zu ähnlichem Effekte: steigt sodann der Zucker- 
spiegel an, so kann - falls die zuckerverwertenden Falktoren in Leber. und Geweben nicht gleichzeitig mitgelitten haben - der zweite Teil der Ambardschen Regel in Kraft tireten.

Wie steht es nun mit den Besserungen und Heilungen der diabetischen Stoffwechselstörung, wie sie manchmal bei Tuber-. kulösen und Krebskranken in Erscheinung tritt? Ist diese auch bloß auf die verschieden abgestufte Konstellation der physiologischen diabetogenen und antidiabetogenen Falztoren zurïckzuführen oder spielen die spezilischen Krankheitsprodukte eine einschneidende Rolle? Für die Tuberkulose ist zunächst festzustellen, daß eine Besserung der Toleranz nur in den seltensten Fiallen zu finden ist. Bei meinem großen Diabetikermateriale konnte ich es nur zweimal beobachten. Das eine Mal handelte es sich um eine fibröse Phthise mit vikarierendem Emphysem und starken pleuroperikardialen Verwachsungen. Die kardiale Insuffizienz beherrschte das Bild und nicht der infektiöse ProzeB; im zweiten Falle bestand eine typische kavernöse Phthise des rechten oberen Lungenlappens béi einem jungen Menschen, der ein halbes Jahr vorher mit 60 Insulineinheiten pro Tag und $80 \mathrm{~g}$ KohJenhydraten zucker- und azetonfrei gemacht werden konnte und dessen Toleranz jetzt bei 10 Einheiten. $150 \mathrm{~g}$ Kohlenhydrate betrug. Allerdings war er unterdes maximal abgemagert.

Allen sieht auch in diesen Besserungen nur einen Spezialfall für die antidiabetische Wirkung der starken Abmagerung. Neuerdings hat Rosenberg aus der Abteilung Umber's eine Anzahl von Krankengeschichten publiziert, aus welchen eine weitgehende Besserung der Zuckerkrankheit bei komplizierender schwerer Phthise hervorgeht. Ihn befriedigt die Erlklärung Allens nicht, sondern er nimmt eine spezifische Beeinflussung des Kohlenhydratstoffwechsels durch den tuberkulösen Prozeß an. Noch weiter geht Lundberg mit der von ihm auch experimentell gestützten Hypothese, daß das verkäste Gewebe tuberkulösen Ursprungs einen insulinartigen Stoff produziert, der im Tierversuche Hypoglykämie und Blutdrucksenkung hervorruft.

Im allgemeinen. möchte ich daran zweifeln, daß bei diesen Fällen ein spezifischer Einfluß des tuberkulösen Gewebes auf den Zuckerverbrauch angenommen werden muß. Ja, ich bin der Ansicht, daß es nur zur Verwickelung der Insulinfrage führen kann, wenn man jede auf irgendeine Weise aus irgendeinem Gewebe extrahierte Substanz, die im Tierversuch zur Erniedrigung des Blutzuckerspiegels führt, als insulinartig oder gar als Parainsulin 
bezeichnet. - Ebenso könnte man das Koffein, das zu einer Blutdrucksteigerung führt, ein Paraadrenalin nennen. Sicher ist die diabetesbessernde Wirkung bei gewissen tuberkulösen Erkrankungen nicht spezifisch, denn eine solche kommt ab und zu auch nach anderen akuten, fieberhaften Krankheiten vor. Ich habe sie zweimal nach Polyserositis gesehen, welche sicherlich nicht tuberkulösen Ursprungs war. Ähnliches berichtete Gläßner nach Grippe.

Auf weniger hypothetischem Boden bewegen wir uns, wenn wir unsere Aufmerksamkeit dem Einflusse der Krebskrankheit auf die diabetische Stoffwechselstörung zuwenden. Es ist eine bekannte Tatsache, claß die karzinomatöse Kachexie eine Heilung der Znckerkrankheit mit sich bringt und zu einer ausgesprochenen Hebung der Kohlenhydrattoleranz führt. Boas war einer der ersten, der auf diese 'Tatsache aufmerksam machte. Wir sehen dies am ehesten bei dem Leberkrebs, welcher ja bei Diabetikern ganz besonders häufig ist. Bisher schien mir eine Herabsetzung des Energie- und Eiweißumsatzes, die mit einer Hypofunktion der endolrinen Drüsen und des vegetativen Nervensystems einhergeht, diese Tatsache genügend zu erlzlären, doch sind neuerdings Versuche auf breitester Basis und von autoritativer Seite veröffentlicht worden, aus denen die gesteigerte Fähigkeit der Krebszellen, Zucker zu zersetzen, mit Sicherheit hervorgeht. Bringt man Krebsgewebe mit einer Dextroselösung von $0,5-1 \%$ zusammen, so sinkt der Zuckergehalt um $30-40 \%$. Nach den aufsehenerregenden Mitteilungen Warburgs und Minamis kann das aus Ratten gewonnene Krebsgewebe 70 mal mehr Milchsäure aus Zucker bilden, als das gesunde Gewebe. Der Tumor zerlegte in 12 Stunden soviel Zucker, als seinem eigenen Gewichte entspricht. Neuschloß wies nach, daß durch die Zugabe von Blausäure dieser Prozeß gehemmt werde, und schließt daraus, daß es sich eigentlich um einen gärungsartigen $V$ organg handle.

Ich will meine Darlegungen im folgenden kurz zusammenfassen:

Diejenigen Faktoren, welche die Spontanheilung der Zuckerkrankheit herbeiführen, stimmen im allgemeinen mit denjenigen überein, welche wir bewußt in der modernen Diabetestherapie zur Anwendung bringen. Die Wiederneubildung, vielleicht Hyperplasie des Pankreasinselapparates, hemmt die Zuckermobilisierung in der Leber und verbessert die Zuckerverwertung in der Peripherie; die 
232 Benedict, Bedingungen für dic Spontanhoilung der Zuekexkrankheit?

Atrophie der Leber und des azinösen Pankreasgewebes nerlangsamt den Eiveißumsatz. Die Hypofunktion der Schilddrïse setzl ebenfalls den Kalorien- und Eiveißbedarf herab and hebt dis: Kohlenhydrattoleranz. Die beiden Kardinalpunkte unserer Diabolestherapie: Insulinzufuhr und Verminderung des Eiveißumsatzes, erreicht dor Organismus so mit eigenen Mitteln. Dor Zuckertoleranz bessernde Einfluß des gesteigerten Zuckerangebotes an die Körperzellen bei Nierenkranken findet seine Analogie in der bewapl goibhton Kollenhydratherapie. All das, was mühevolle Untersuchungen in Jahrzehnten als heilsam für den Diabetiker erkannten, wermag der Organismus in gewissen Fällen selbst in die Woge zu leilen, and diese. Erkenntnis, gleichzeitig eine Bestätigung des allon Spruches: „Nalura sanat, medicus curat", kann uns gleichzeitig fïr die 'T'ulsache entschädigen, daß die "Spontanheilung" des Diabetes im konlwoln F'alle? dem Kranken kein sicheres Heil, dem Arse keine reine Bo/rioligung gewälurt.

\section{Lilorallur.}

Allen, Med. Clin. of North-America. Now-York. Bal. B. Tour. of metabolic Research. Bd. 1. - Ambard, Schmid u. Arnolyjovich, Prosso mód. 1924. - Benedict, H., u. Roth, Ztsohr. f. klin. Med. BAl. 71. .... Bernard, Cl., Vorlesungen über Diabetes. Berlin 1878, - Boas, Borl. klin. Wodh, $1904 .-\cdots$ Bouchardat, De la Glycosurie du Diabèto sucré. Puris 1875. (Zit. nuch Falta.) - Düring, Ursache und Heilung des Diabotes nellitas. Hammerer 1868. - Eppinger, Falta u. Rudinger, Ztschr. E. klin. Mol. Bul. 66. -..Falta, Die Mehlfrüchtekur bei Diabetes mellitıs. Wion 1920. …. Fritulmann u. Goltesmann, Proc. of the Soc. f. exper. biol, Bd, 18. -..- Ciluessn'r, Wier. klin. Woch. 1906. - Herxheimer, Zentralbl. f. Pathol. u, path. Anat. Festschrift f. M. B. Schmidt. - Hoppe-Seyler, Münch. mod. Woch. Ju. 71. 1924. Dtsch. Ztschr. f. Jim. Med. 145/46. (Literatur äber Insulrogenoration.) - Kolisch, Med. Klin. 1903. - Kraus. E. J., Virchows Arohiv. Bd. 247. - Ledine, Le Diabìte sucré. Paris 1909, …- Lundberg, Actia meil. scandinar. Supl. Bd. 7. -- Maignon, Comptes rendus des séuncess de lia soe. des Biologie. Bd. 86. - Naunyn, Der Diabetes mellitus in Nolhuagrels Spez. Path. u. Ther. Bd. VII. 1. Teil. - Neuschloss, Klin. Wooh. Jg. 3, Noorden, Die Zuckerkrankbeit. 5. Aufl. Wien 1910. — Petr:n, Verhandl. d. d. Kongr: f. inn. Med. 1922. - Rhodenburg. Endoerinologry. Bal. (1.-Rosenberg, Klin. Woch. Jg. IV. 4. - Schiltenheln, Münch. mod. Wodh. 1926. 73. Jg. S. 1641. - Warburg-Minami, Bioch. Ztschr. Bd. 142. 